J. C. BECKER, Abhandlungen aus dem Grenzgebiete der Mathematik und Philosophie. Zürich, $18 \% 0$.

ANs ARBor, August 1, 1891.

Alexander Ziwet.

\title{
PICARD'S DEMONSTRATION OF THE GENERAL THEOREM UPON THE EXISTENCE OF INTEGRALS OF ORDINARY DIFFERENTIAL EQUATIONS.
}

TRANSLATED BY DR. THOMAS S. FISKE.

THE cardinal proposition in the theory of algebraic equations, that every such equation has a root, holds a place in mathematical theory no more important than the corresponding proposition in the theory of differential equations, that every differential equation defines a function expressible by means of a convergent series. This proposition was originally established by Cauchy, and was introduced, with a somewhat simplified demonstration, by Briot and Bouquet in their treatise on doubly periodic functions.* A new demonstration remarkable for its simplicity and brevity has been published by M. Emile Picard in the Bulletin de la Société Mathématique de France for March, $\uparrow$ and reproduced on account of its striking character in the Nouvelles Annales a'es Mathématiques for May. This demonstration requires no auxiliary propositions, and depends upon no preceding part of the theory, except the simple consideration, that any ordinary differential equation is equivalent to a set of simultaneous equations of the first order.t The following is a translation of Picard's demonstration.

1. Consider the system of $n$ equations of the first order

$$
\begin{aligned}
& \frac{d u}{d x}=f_{1}(x, u, v, \ldots, w), \\
& \frac{d v}{d x}=f_{2}(x, u, v, \ldots, w), \\
& \dot{d w} \cdot \cdot . \cdot . . . ., \\
& \frac{d x}{d x}=f_{n}(x, u, v, \ldots, w),
\end{aligned}
$$

* Théorie des fonctions elliptiques, p. 325.

JoRDAN. C'ours d'analyse, vol. III., p. 87.

+ Bulletin de la Société Mathématique de France, Vol. XIX., p. 61.

‡ JoRdan. Cours d'analyse, vol. III., p. 4. 
in which the functions $f$ are continuous real functions of the real quantities $x, u, v, \ldots, w$ in the neighborhood of $x_{0}, u_{0}$, $v_{0}, \ldots, w_{0}$, and have determinate values as long as $x, u, v$, ..., $w$ remain within the respective intervals

$$
\begin{aligned}
& \left(x_{0}-a, x_{0}+a\right), \\
& \left(u_{o}-b, u_{0}+b\right), \\
& \left(v_{o}-b, v_{o}+b\right), \\
& \cdot \cdot \cdot \cdot \cdot \cdot \cdot \\
& \left(w_{o}-b, w_{\circ}+b\right),
\end{aligned}
$$

$a$ and $b$ denoting two positive magnitudes.

Suppose that $n$ positive quantities $A, B, \ldots, L$ can be determined in such a manner that

$$
\begin{aligned}
& \left|f\left(x, u^{\prime}, v^{\prime}, \ldots, w^{\prime}\right)-f(x, u, v, \ldots, w)\right| \\
& \quad<A\left|u^{\prime}-u\right|+B\left|v^{\prime}-v\right|+\ldots+L\left|w^{\prime}-w\right|,
\end{aligned}
$$

in which $|\alpha|$ denotes as usual the absolute value of $\alpha$, and $x, u, v, \ldots, w$ are contained in the indicated intervals. This will evidently be the case when the functions $f$ have finite partial derivatives with respect to $u, v, \ldots, w$.

Starting with these very general hypotheses we will demonstrate that there exist functions $u, v, \ldots, w$ of $x$, continuous in the neighborhood of $x_{o}$, satisfying the given differential equations, and reducing, for $x=x_{0}$, respectively to $u_{o}, v_{o}$, ..., $w_{\circ}$.

2. We proceed by successive approximations. Taking first the system

$$
\begin{aligned}
& \frac{d u_{1}}{d x}=f_{1}\left(x, u_{o}, v_{o}, \ldots, w_{o}\right), \\
& \cdot . \cdot . \cdot . \cdot . \cdot . \cdot . \\
& \frac{d w_{1}}{d x}=f_{n}\left(x, u_{o}, v_{\lrcorner}, \ldots, w_{o}\right),
\end{aligned}
$$

we obtain by quadratures the functions $u_{1}, v_{1}, \ldots, w_{1}$, determining them in such a manner that they take for $x_{0}$ the values $u_{o}, v_{o}, \ldots, w_{0}$. Forming then the equations

$$
\begin{aligned}
& \frac{d u_{2}}{d x}=f_{1}\left(x, u_{1}, v_{1}, \ldots, w_{1}\right), \\
& \cdot \cdot \cdot \cdot \cdot . \cdot . \cdot . \cdot, \\
& \frac{d w_{2}}{d x}=f_{n}\left(x, u_{1}, v_{1}, \ldots, w_{1}\right),
\end{aligned}
$$


we determine $u_{2}, v_{2}, \ldots, w_{2}$ by the condition that they take for $x_{o}$ the values $u_{o}, v_{o}, \ldots, w_{o}$, respectively. We continue this process indefinitely, the functions $u_{m}, v_{m}, \ldots, w_{m}$ being connected with the preceding $u_{m-1}, v_{m-1}, \ldots, w_{m-1}$ by the relations

$$
\begin{aligned}
& \frac{d u_{m}}{d x}=f_{1}\left(x, u_{m-1}, v_{m-1}, \ldots, w_{m-1}\right) \\
& \frac{d w_{m}}{d x}=f_{n}\left(x, u_{m-1}, v_{m-1}, \ldots, w_{m-1}\right) \text {, }
\end{aligned}
$$

and, for $x=x_{o}$, satisfying the equations

$$
u_{m}=u_{o}, v_{m}=v_{o}, \ldots, w_{m}=w_{\circ} \text {. }
$$

We will now prove that when $m$ increases indefinitely, $u_{m}$, $v_{m}, \ldots, w_{m}$ tend toward limits which represent the integrals sought provided that $x$ remains sufficiently near $x_{0}$.

Let $M$ be the maximum absolute value of the functions $f$ when the variables upon which they depend remain between the indicated limits. Denote by $\rho$ a quantity at most equal to $a$. If now $x$ remains in the interval

we have

$$
\left(x_{o}-\rho, x_{o}+\rho\right) \text {, }
$$

$$
\left|u_{1}-u_{0}\right|<M \rho, \ldots,\left|w_{1}-w_{0}\right|<M \rho .
$$

Hence, provided $M \rho<b$, the quantities $u_{1}, v_{1}, \ldots, w_{1}$ remain within the desired limits, and it is evident that the same is true of all the other sets of values $u, v, \ldots, w$. Denoting by $\delta$ a quantity at most equal to $\rho$, suppose that $x$ remains in the interval

and write

$$
\left(x_{0}-\delta, x_{0}+\delta\right)
$$

$$
u_{m}-u_{m-1},=U_{m}, \ldots, w_{m}-w_{m-1}=W_{m} ;
$$

we have, placing $m=2,3, \ldots, \infty$,

$$
\begin{aligned}
& \frac{d U_{m}}{d x}=f_{1}\left(x, u_{m-1}, \ldots, w_{m-1}\right)-f_{1}\left(x, u_{m-2}, \ldots, w_{m-2}\right), \\
& \cdot . \cdot . \cdot . \cdot . \cdot . \cdot . \cdot . \cdot . \cdot . \cdot, \\
& \frac{d W_{m}}{d x}=f_{n}\left(x, u_{m-1}, \ldots, w_{m-1}\right)-f_{n}\left(x, u_{m-2}, \ldots, w_{m-2}\right) .
\end{aligned}
$$


Since

$$
\left|U_{1}\right|<M \delta, \ldots,\left|W_{1}\right|<M \delta,
$$

the preceding equations, for $m=2$, show that $\left|U_{2}\right|,\left|V_{2}\right|$, $\ldots,\left|W_{2}\right|$ are less than

$$
(A+B+\ldots+L) M \delta^{2} .
$$

Continuing step by step it may be shown that $\left|U_{m}\right|, \ldots$, $\left|W_{m}\right|$ are less than

$$
M \delta(A+B+\ldots+L)^{m-1} \delta^{m-1} .
$$

Now since

$$
u_{m}=u_{o}+U_{1}+U_{2}+\ldots+V_{m},
$$

$u_{m}$, and also $v_{m}, \ldots w_{m}$, will tend toward finite limits if

$$
(A+B+\ldots+L) \delta<1 \text {. }
$$

This condition will be fulfilled by making $\delta$ sufficiently small.

We see then that $u_{m}, v_{m}, \ldots, w_{m}$ tend toward determinate limits, $u, v, \ldots, w$, which are continuous functions of $x$ in the interval

$$
\left(x_{o}-\delta, x_{o}+\delta\right),
$$

$\delta$ being the smallest of the three quantities

$$
a, \frac{b}{M}, \frac{1}{A+B+\ldots+L},
$$

and that $u, v, \ldots, w$ are represented by series which converge after the manner of a decreasing geometrical progression.

Moreover we have

$$
u_{m}=\int_{x_{0}}^{x} f_{1}\left(x, u_{m-1}, \ldots, w_{m-1}\right) d x+u_{o},
$$

and, since $u_{m}, v_{m}, \ldots, w_{m}$ differ from their limits as little as we please, whatever the value of $x$ in the indicated interval, when $m$ is sufficiently great, we have in the limit

$$
u=\int_{x_{0}}^{x} f_{1}(x, u, v, \ldots, w) d x+u_{o}
$$


CALCUL DES PROBABILITÉS.

Hence

$$
\frac{d u}{d x}=f_{1}(x, u, v, \ldots, w) .
$$

Similar results hold for the other functions. The functions $u, v, \ldots, w$ are consequently the functions sought.

CALOUL DES PROBABILITES. Par J. Bertrand, de l'Académie Française, Secrétaire perpétriel de l'Académie des Sciences. Paris, Gauthier-Villars, 1889. 8vo., LVII $+332 \mathrm{pp}$.

THene is possibly no branch of mathematics at once so interesting, so bewildering, and of so great practical importance as the theory of probability. Its history reveals both the wonders that can be accomplished and the bounds that cannot be transcended by mathematical science. It is the link between rigid deduction and the vast field of inductive science. A complete theory of probability would be a complete theory of the formation of belief. It is certainly a pity then, that, to quote M. Bertrand, "one cannot well understand the calculus of probabilities without having read La Place's work," and that "one cannot read La Place's work without having prepared himself for it by the most profound mathematical studies."

Though not otherwise is thorough knowledge to be gained, yet an exceedingly useful amount of knowledge is to be had without such effort. In fact, M. Bertrand's forty odd pages of preface on "The Laws of Chance" give an insight into the theory without the use of so much as a single algebraic symbol.

Listen to this reductio ad absurdum of Bernoulli's theory of moral expectation:

'6 'If I win,' says poor Peter, proposing a game of cards to Paul, 'you must pay three francs for my dinner.' ' Meal for meal,' replies Paul, 'you should pay twenty francs in case you lose, for that is the price of my supper.' 'If I lose twenty francs,' cries Peter, frightened out of his wits, 'I cannot dine to-morrow: without coming to that you might lose a thousand; put them up against my twenty. According to Daniel Bernoulli, you will still have the advantage." "'

Even more complete is the upsetting of Condorcet's calculation as to the probability of the sun's rising.

"Paul would wager that the sun rises to-morrow. The theory fixes the stakes. Paul shall receive a franc if the sun rises and will give a million if it fails to do so. Peter accepts 